\title{
Microsurgical and Endoscope Assisted Resection of a Right Intracanalicular Vestibular Schwannoma Two-Dimensional Operative Video
}

\author{
Chun-Yu Cheng ${ }^{1,2}$ Zeeshan Qazi ${ }^{2} \quad$ Laligam N. Sekhar ${ }^{2}$ \\ ${ }^{1}$ Department of Neurosurgery, Chang Gung Memorial Hospital, \\ Chiayi, College of Medicine, Chang Gung University, Taiwan, Taiwan \\ Address for correspondence Chun-Yu Cheng, MD, Department of \\ Neurosurgery, University of Washington, Harborview Medical Center, \\ ${ }^{2}$ Department of Neurosurgery, University of Washington, Seattle, \\ 325 9th Avenue, P.O. Box 359924 Seattle, WA 98104, United States \\ Washington, United States \\ (e-mail: chuntoo@gmail.com).
}

J Neurol Surg B 2019;80(suppl S3):S288-S289.

\begin{abstract}
A 36-year-old lady presented with tinnitus and hearing loss for 1 year which was progressively worsening. A hearing test revealed pure tone average (PTA) between 48 to $65 \mathrm{~dB}$ and speech discrimination of $56 \%$ at $95 \mathrm{~dB}$. Brain magnetic resonance imaging (MRI) showed a right vestibular schwannoma $5 \times 8 \mathrm{~mm}$ (-Fig. 1) which extended far laterally to the fundus of internal auditory canal (IAC). A translabyrinthine approach was suggested by another neurosurgeon/neurotologist team, but the patient decided to undergo operation by retrosigmoid approach with attempted hearing preservation. She underwent a right retrosigmoid craniotomy, craniectomy, and mastoidectomy with far lateral approach. We performed petrous transcanalicular microsurgical approach with the assistance of neuroendoscope. Intraoperatively, the internal auditory artery was looping into the IAC between cranial nerves VII and VIII, and coming out inferiorly. The IAC was opened by the diamond drill, ultrasonic bone curette, and fine rongeurs. The tumor was grayish in color with filling the lateral aspect of the IAC. After circumferential dissection of the tumor capsule, the tumor was

Keywords

- microsurgical

- endoscopic

- intracanalicular

- vestibular schwannoma

- retrosigmoid approach removed completely. It was arising from the inferior vestibular nerve which was stretched. The patient had vertigo and nausea postoperatively but it is steadily improving. Her hearing test has improved to a PTA of $22 \mathrm{~dB}$ and speech discrimination of $100 \%$ at $70 \mathrm{~dB}$ at 6 weeks. The postoperative MRI showed total resection.

This two-dimensional video shows the technical nuances of microsurgical retrosigmoid approach and endoscopic assisted resection of an intracanalicular vestibular schwannoma and the value of attempting hearing preservation in all vestibular schwannomas (- Fig. 2). The link to the video can be found at: https://youtu.be/KHrO_iDI2tw.
\end{abstract}

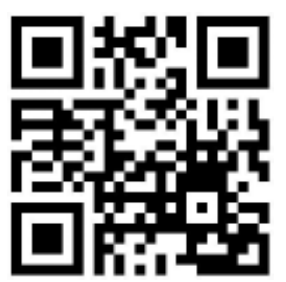

received

April 23, 2018 accepted after revision November 11, 2018 published online January 24, 2019

www.thieme.com/skullbasevideos

www.thieme.com/jnlsbvideos

DOI https://doi.org/

10.1055/s-0038-1676840. ISSN 2193-6331. (c) 2019 Georg Thieme Verlag KG Stuttgart · New York
License terms

(c) $(1) \$$ 
Statement Regarding Patient Consent

Informed consent was obtained from the patient prior to the surgery that included videotaping of the procedure and its distribution for educational purposes. All relevant patient identifiers have also been removed from the video and accompanying radiology slides.

\section{Disclosure of Funding}

None.

\section{Conflict of Interest}

None declared.
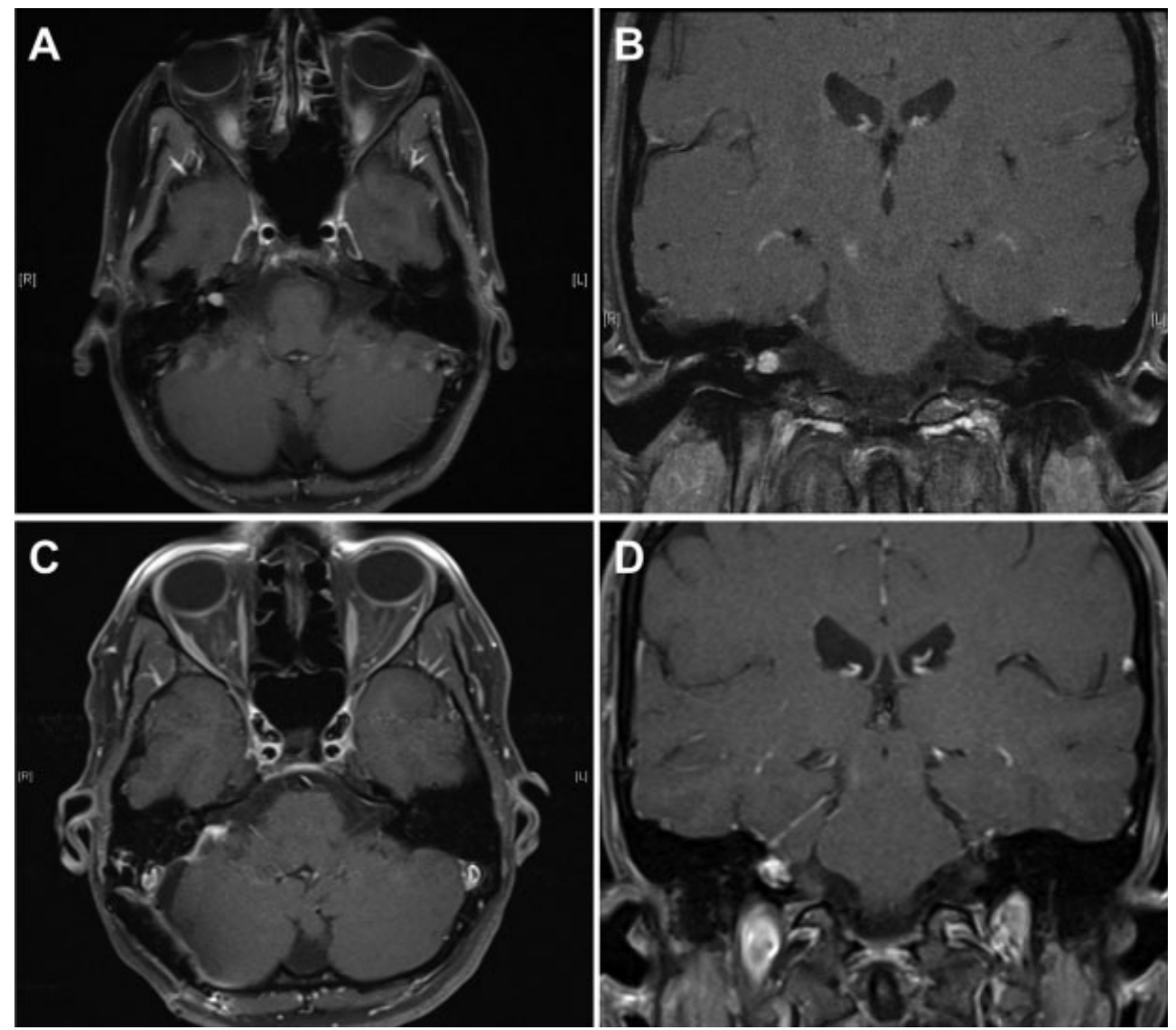

Fig. 1 Preoperative brain magnetic resonance imaging (MRI) with gadolinium contrast axial (A) and coronal (B) view showing a right intracanalicular tumor which extended far laterally to the fundus. At 6 weeks of follow-up, postoperative axial (C) and coronal (D) brain MRI with gadolinium contrast demonstrating complete resection of the lesion and scaring of the internal auditory canal.
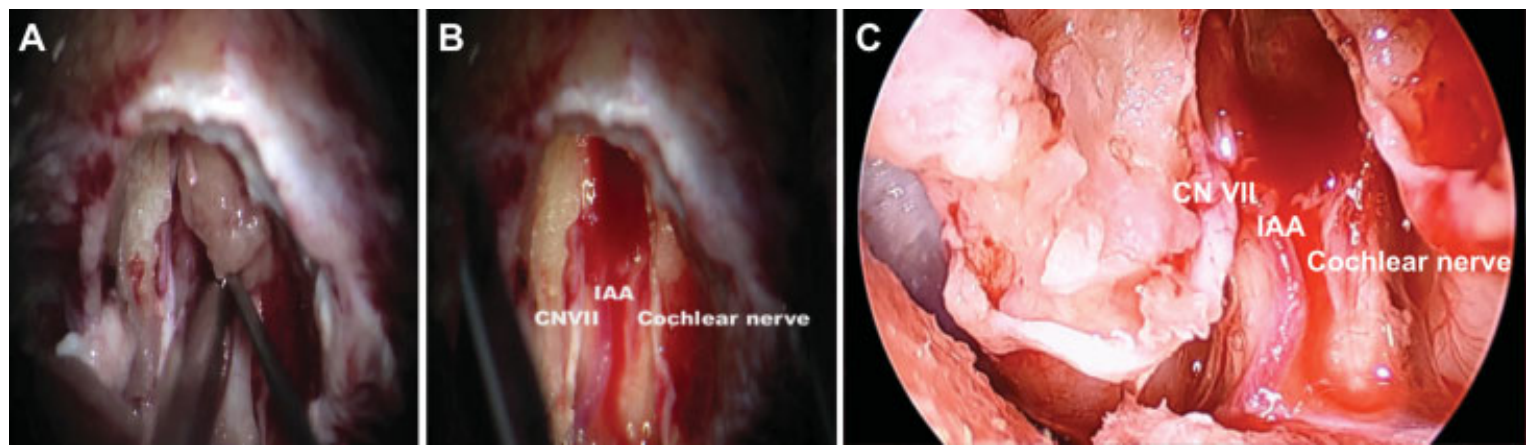

Fig. 2 Intraoperative resection of the tumor (A) transcancalicular dissection of the tumor; Complete resection of the tumor is achieved from microscopic view (B) from the endoscopic view (C). CN, cranial nerve; IAA, internal auditory artery. 